\title{
Structure of the Nerve Myelin Membrane: Proof of the Low-resolution Profile
}

\author{
Allen E. Blaurock $\dagger$ \\ Physics Department and the Biophysics Division of the Institute of Science and \\ Technology, University of Michigan, Ann Arbor, Mich., U.S.A.
}

(Received 15 September 1970, and in revised form 16 November 1970)

\begin{abstract}
In analyzing the low-angle X-ray diffraction patterns recorded from the normal and swollen myelin in frog sciatic nerve, a precise value is found for the centerto-center distance between the pair of membranes constituting the repeating structure in the myelin. The previously reported large increase in period when the nerve is put in hypotonic Ringer's solution is shown to be accompanied by a small decrease in the center-to-center distance of the membranes within one pair. Thus both a large amplitude swelling at the extracellular interface between membrane pairs and a moderate one at the cytoplasmic interface within the pair are observed. The two kinds of swelling-cytoplasmic and extracellular-are sufficient to demonstrate crystallographically the correctness of the bilayer profile for a single myelin membrane. The existence of a lipid bilayer within the membrane is confirmed by the scale of absolute electron density derived from the swelling experiments, and hydrated surface layers of protein are postulated.
\end{abstract}

\section{Introduction}

Myelinated nerve is a desirable specimen for X-ray diffraction analysis of a membrane structure because the sharp low-angle reflections, which arise from the many closely packed pairs of membrane in each of the myelin sheaths, are clearly distinguished from diffraction arising elsewhere in the nerve. Also, because the myelin membrane, which is a specialized area of plasma membrane (Geren, 1954; Robertson, 1958), is generally well oriented, diffraction indicating structure along the membrane surface can be recognized, e.g. oriented diffraction identified with the side-to-side packing of lipid hydrocarbon chains shows that they tend to be oriented perpendicular to the membrane surface (Schmitt, Bear \& Clark, 1935).

Yet to observe the sharp, regularly spaced-Bragg's law-reflections presents difficulties in interpreting the low-angle pattern. For, while a dispersion of single pairs of membranes will give a continuous curve of diffraction intensity (Wilkins, Blaurock \& Engelman, 1971), the regular stacking of a number of membrane pairs in myelin results in sharp reflections, the intensities of which sample the continuous curve for a single pair only at widely spaced points. However, myelin swelling in hypotonic Ringer's solution, when broad fluid layers separate the extracellular surfaces of adjacent membrane pairs (Robertson, 1958), offers the opportunity to record the valuable intervening intensity data.

$\dagger$ Present address: Cardiovascular Research Institute, University of California, San Francisco, Calif. 94122, U.S.A. 
Finean \& Burge (1963) and Moody (1963) showed that, for sciatic nerve myelin swollen to varying degrees in hypotonic Ringer's solution (Finean \& Millington, 1957), a simple curve is outlined by the diffraction intensities. This curve, which was taken to confirm the assumption of constant membrane pair structure, was used to interpret the normal pattern. The correct phasing of the normal pattern will give the membrane profile, that is, the average cross-sectional electron density curve determined by the arrangement of the constituent lipids, protein and water.

I have observed a second kind of myelin swelling and shrinking, also reversible, which is at the cytoplasmic interface between membrane pairs (Blaurock, 1967); this cytoplasmic swelling occurs in general when the concentration of the Ringer's or other salt solution bathing frog sciatic nerve is varied. Thus both a small amplitude cytoplasmic and a large amplitude extracellular swelling occur when a nerve is immersed in hypotonic Ringer's solution whereas only cytoplasmic swelling has been observed when the Ringer's or other salt solution is made hypertonic. Analysis of the cytoplasmic swelling removes ambiguities (Moody, 1963) remaining after analyzing the extracellular swelling; in consequence the bilayer structure of the myelin membrane is demonstrated crystallographically. By varying the concentration of sucrose solution, in which myelin swells as in water (Worthington \& Blaurock, 1969a), an absolute scale of electron density is found. This scale confirms that the membrane contains a lipid bilayer. The importance of salt ions in maintaining the normal myclin structure has been demonstrated (Worthington \& Blaurock, 1969a); their presence is now shown directly on the profile.

\section{Methods}

Myelin sheath was swelled by soaking a freshly dissected frog sciatic nerve in distilled water at $4^{\circ} \mathrm{C}$ for a period of 9 to $30 \mathrm{hr}$. To tost the offoct of sucroso or of various salt solutions, a sciatic nerve previously immersed in water was soaked in the test solution at $4^{\circ} \mathrm{C}$ for at least $12 \mathrm{hr}$ (Worthington \& Blaurock, 1969a). To compare $\mathrm{LiCl}$ with $\mathrm{CsCl}$, freshly dissected sciatic nerve was soaked in a 0.125 or $0.25 \mathrm{M}$ solution at room temperature for at least $24 \mathrm{hr}$ (Blaurock, 1967). All specimens were exposed at room temperature as described (Blaurock \& Worthington, 1969). X-ray exposures lasting from 1 to $24 \mathrm{hr}$ recorded the pattern to a Bragg's law spacing of about $30 \AA$ at a density suitable for measuring. The repeat distance of myelin membrane pairs, $D$, was obtained directly from the measured regular spacing of the reflections on the film. Integrated intensities of the reflections were measured on a radial densitometer trace. The measured intensities of the set of reflections from one pattern were corrected by multiplying each intensity, $I_{h}$, and its order number, $h$, together (Blaurock \& Worthington, 1966) to obtain $F_{h}^{2}=\alpha \cdot I_{h} \cdot h$, where $\alpha$ is the scale factor.

To compare sets of corrected reflection intensities from swollen myelin, each set was scaled (Blaurock, 1967) by choosing $\alpha$ to make the sum $\alpha \sum_{h=1}^{H} I_{h} \cdot h=\sum_{h=1}^{H} F_{h}^{2}$ equal to the ratio $D / 171 \AA \dagger(171 \AA$ is the normal period); $H$ is generally the largest integer less than or equal to $D / 28 \AA$. In each case the Patterson function was computed using the set of corrected reflection intensities and scaled to be equal to unity at the origin of the Patterson function. A scaled Fourier synthesis was computed by using the square roots, $F_{h}$, of the set of corrected reflection intensities and dividing the synthesis at each point by the ratio

$\dagger$ The mathematical expression is properly $\sum_{h=-\infty}^{+\infty} F_{h}^{2}=D / 171 \mathrm{~A}$, but in practice $F_{0}^{2}$ is not observed, and $|h|$ is limited to a finite range. A mathematical consideration of the consequent errors has been given (Blaurook, 1967). The errors in the present study are taken up in seotion (d) of the Disoussion. 
$171 \AA / D$ to obtain a profile which could be directly compared (Blaurock, 1967) to other swollen myelin profiles.

\section{Analysis}

\section{(a) Myelin swelling in water and in sucrose solutions}

For nerve in water or in a solution $0.24,0.82$ or $1.80 \mathrm{~m}$ in sucrose the myelin period, $D$, increased from the normal $171 \AA$ to a value in excess of $250 \AA$ (Worthington \& Blaurock, 1969a). In each of these cases of swelling, the Bragg's law low-angle reflections were symmetrically broadened, the reflection half-width increasing regularly with $h$ until, in some cases, higher order reflections merged to form a broad band of film density. Thus, since there generally was considerable disorder in the spacing of the membranes in swollen myelin, $D$ is an average period.

The swelling in water confirms the X-ray observations of Finean \& Millington (1957), of Moody (1963), and of Finean \& Burge (1963). In addition, our results (Worthington \& Blaurock, 1969a) show clearly that the swelling was not osmotic since, on the one hand, it occurred in water and in solutions of osmolarity both comparable to Ringer's $(0 \cdot 24 \mathrm{M}$-sucrose) and much higher (1.80 $\mathrm{M}$-sucrose) and, on the other hand, a solution

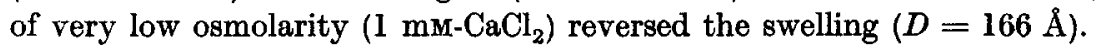

We have shown (Worthington \& Blaurock, $1969 b$ ) that the square roots, $F_{h}$, of the corrected, scaled myelin reflection intensities from nerves in water and dilute salt solutions outline a simple curve, denoted $F_{1}$, with two main peaks: regions $B$ and $\mathrm{C}$ in Figure 1(a). Scaled sets of the square roots, $F_{n}$, of corrected reflection intensities for nerve in $\mathbf{0 . 2 4} \mathbf{M}$-sucrose solutions were shown (Worthington \& Blaurock, $1969 b$ ) to outline a simple, smooth $F_{I}$ curve (Figure $1(b)$ ) closely similar to the one for swelling in water. The single set of corrected reflection intensities for nerve in 0.82 M-sucrose $(D=359 \AA)$ and the set for nerve in $1.80 \mathrm{M}$-sucrose $(D=426 \AA)$ also fit on similar curves (Fig. 1(c) and see Blaurock, 1967). Thus for myelin swollen to various large periods in water and in sucrose solutions, the reflection intensities outline two broad peaks.

The existence of the $F_{I}$ curve in Figure l(a) confirms the assumption that the membrane pair structure is independent of the degree of (extracellular) swelling in water; similarly for swelling in $0.24 \mathrm{M}$-sucrose. Equivalently, a constant structure is indicated by the regular features of the Patterson functions (see below).

The observation that the curve apparent for swollen myelin lies close to the reflection intensities from myelin in Ringer's solution (Figure 1(a)) indicates that there are, in swollen myelin, broad fluid layers separating each pair of nearly normal myelin membranes (Geren, 1954) from adjacent pairs. This conclusion is in agreement with the electron microscope observation (Robertson, 1958) that large fluid layers separate the extracellular surfaces of pairs of myelin membranes when frog sciatic nerve is put in hypotonic Ringer's solution.

It is noted that sets of measured reflection intensities, $I_{h}$, from frog and rat sciatic myelin swollen to varying degrees in hypotonic Ringer's solution were fitted by Finean \& Burge (1963) to a simple curve with two broad peaks. When their intensities are corrected-multiplying $I_{n}$ by $h$-one obtains a curve closely similar to the square of the curve in Figure 1(a). For sets of swollen frog sciatic myelin reflection intensitiesuncorrected and scaled by making $\sum I_{h}$ constant-Moody (1963) obtained a curve similar to that of Finean \& Burge. 

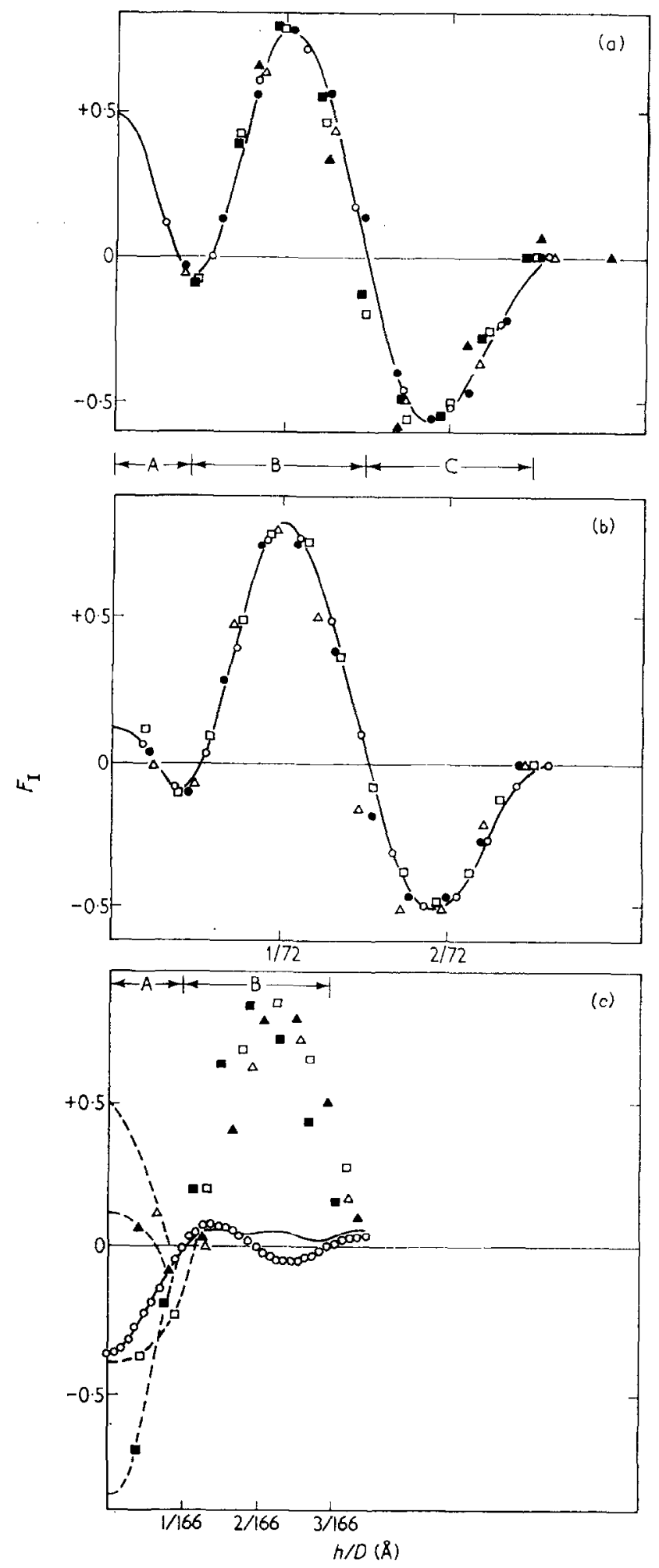

Fíg 1. 


\section{(b) Patterson function analysis}

The membrane profile is the average electron density curve along a line perpendicular to the membrane. A bilayer membrane profile is a curve with two electron-dense peaks separated by a trough of low electron density (Wilkins et al., 1971). For a bilayer membrane profile there will be a Patterson function correlation, i.e. a peak similar to the origin peak, at a distance equal to the distance, $S$, separating the center of one membrane from the center of the other membrane in a pair (Blaurock, 1967). A pair of symmetric membranes will give a pair-correlation peak with the same half width, but only half as high, as the origin peak: as the membrane profile asymmetry increases, the height will decrease.

The Patterson functions computed without assumption from the swollen myelin patterns (see Fig. 2(a) for nerve in water) all show similar features: the largest peak at the origin; a trough generally centered at $24 \AA$; a peak near $70 \AA$ (see Table 1 ); and for water and $1 \mathrm{~mm}^{-\mathrm{CaCl}_{2}}$, a low peak near $40 \AA$ (Fig. 2(a) and (b)) which becomes a shoulder in $0.24 \mathrm{M}$-sucrose and is altogether absent for 0.82 and $1.80 \mathrm{M}$-sucrose. In each Patterson function there is a peak centered near $70 \AA$; as can be seen in Table 1, the half-width of the $70 \AA$ peak is the same as, while its height is abouthalf $\dagger$, that of the origin peak. The peak near $70 \AA$ has been identified with a pair-correlation peak as follows.

Figure 2(a) shows the Patterson function for nerve in water. Superimposed (open circles) is an origin-like peak plotted by shifting the origin peak out to $72 \AA$ and dividing the height at every point by two. The origin-like peak fits well over the range from 40 to $110 \AA$. A similar result holds for nerve in the various sucrose solutions. That the $70 \AA$ peak has nearly the same half-width as the origin peak, even though this width changes with the sucrose concentration (see Table 1), confirms a relationship between the two peaks and makes it reasonable to identify the $70 \AA$ peak with the pair-correlation peak of bilayer membranes in each case. Making this identification in order to interpret the Patterson functions, one finds that the center-to-center separation of the pair of membranes in swollen myelin is from 71 to $78 \AA$ ( $S$ in Table 1 ) and, since the height of the 71 to $78 \AA$ peak is generally about $+0 \cdot 5$, that the single membrane profile is largely symmetric.

$\dagger$ Once again; omitting $F_{0}^{2}$ from the Patterson introduces an error. In the present study this omission introduces only a minor error into the ratio of the $70 \AA$ peak to the origin peak (see section (d) of the Discussion).

Fic. 1. The Fourier transform, $F_{I}$, derived from extracellular swelling, when the crystallo. graphic origin of the membrane pair is at the cytoplasmic interface. $H_{h}^{\prime}$ is plotted versus $h / D$.

(a) Swelling in water and dilute salt solutions compared to the normal pattern: (1) in water, $D=252 \AA(O) ;(2)$ in water, $D=342 \AA$ (O); (3) in $10 \mathrm{~mm}-\mathrm{NaCl}, D=294 \AA$ (四); (4) in $10 \mathrm{~mm}-\mathrm{KCl}$, $D=288 \AA(\square)$ (order 1 not recorded for (2), (3) and (4)); (5) in $1 \mathrm{~mm}-\mathrm{CaCl}_{\mathrm{a}}, D=166 \AA(\Delta)$; (6) in Ringer's solution, $D=171 \AA(\Delta)$. The solid line shows the mathematically reconstructed (Blaurock, 1967) Fourier transform best fitting the data for (1) and (5).

(b) Swelling in 0.24 M-sucrose solutions: (1) $D=293 \AA$ ( $\triangle$ ); (2) $D=388 \AA$ (O); (3) including $4 \mathrm{~mm}-\mathrm{NaCl}, D=373 \AA(\square) ;(4)$ including $4 \mathrm{mM}-\mathrm{KCl}, D=326 \AA$ (O). The solid line shows the reconstructed (Blaurock, 1967) Fourier transform best fitting the data for (2) and (4).

(c) Swelling in various sucrose solutions. The Fourier transforms are shown by the dashed curves: (1) in water, $D=252 \AA(\triangle) ;(2)$ in $0.24 \mathrm{M}$-sucrose, $D=388 \AA(\Delta) ;(3)$ in $0.82 \mathrm{M}$-sucrose, $D=359 \AA$ ( $\square$ ) (curve fitted by hand); (4) in $1.80 \mathrm{M}$-sucrose, $D=426 \AA$ (m) (curve fitted by hand). The solid curve is the difference, subtracting the solid curve in (a) from the solid curve in (b); the open circles (O) show the predicted form of this difference, i.e. the function $\sin (\chi) / \chi$ where $\chi=[\pi \cdot 166 \AA \cdot(h / D)]$. 


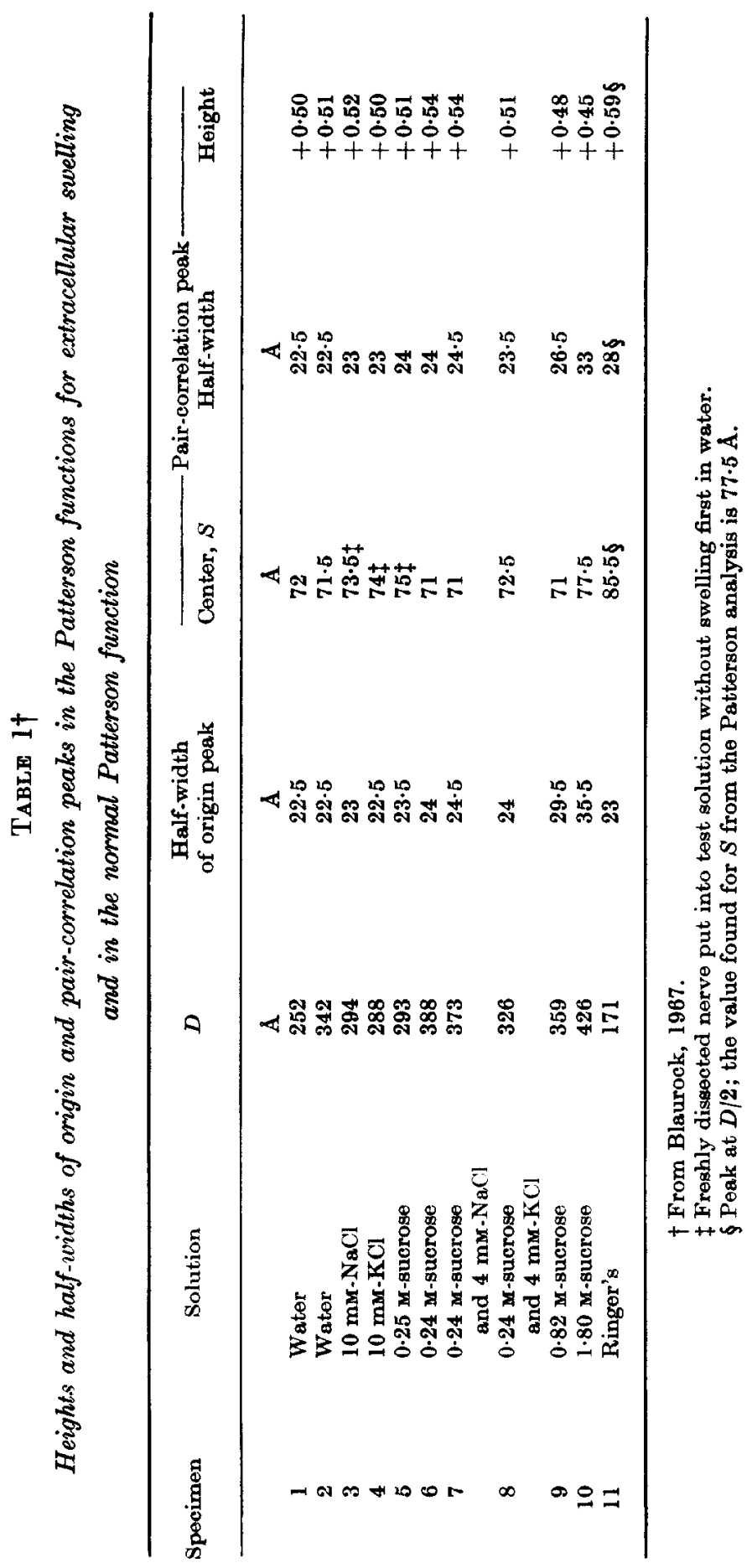




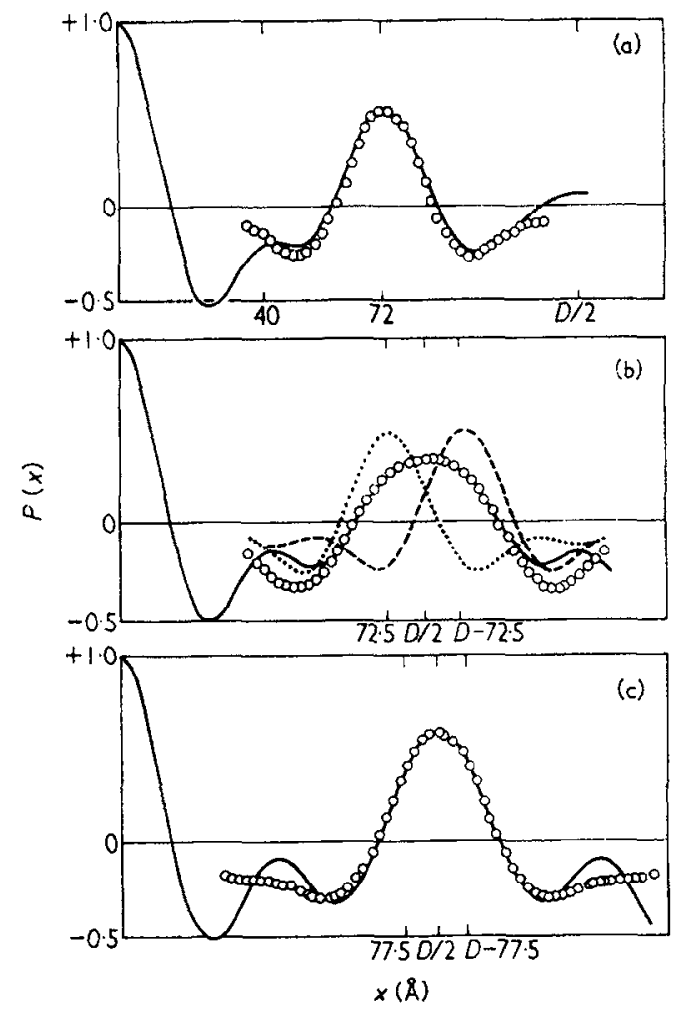

Fig. 2. The Patterson functions, $P$, for the two kinds of myelin swelling. $P(\chi)$ is plotted versus $\chi$.

(a) The Patterson function for nerve in water is shown out to $D / 2(126 \mathrm{~A})$ by the solid curve; the remainder (not shown) of the Patterson function to the right of $D / 2$ is the mirror image of the half-period shown. The open circles (O) show an origin-like peak, i.e. the origin-peak ordinates divided by two, centered at $72 \AA$. The good fit confirms the identification of the $72 \mathrm{~A}$ peak with the pair-correlation peak expected for bilayer membrenes (see text).

(b) The Patterson function for nerve in $1 \mathrm{~mm}-\mathrm{CaCl}_{2}, D=166 \AA$, is shown by the solid curve. The dotted and dashed lines show two origin-like peaks centered at $72.5 \AA$ and at $D-72.5 \AA$ $(93.5 \mathrm{~A})$ respectively, and the open ciroles $(O)$ show the algebraic sum of these two peaks. The good fit of this sum shows the half-period peak to be the resultant of two pair-correlation peaks which are not resolved.

(c) The normal Patterson function, $D=171 \AA$, is shown by the solid curve. The open circles (O) show the algebraic sum of two origin-like peaks centered at $77.5 \AA$ and at $93 \cdot 5 \AA(D-77 \cdot 5 \AA)$. The good fit shows the half-period peak once again to be the sum of two pair-correlation peaks which are not resolved. Note that the right origin-like peak (not shown) is found to be centered at the samo distance, $93.5 \mathrm{~A}$, as the dottod peak in (b).

The good fit of the $1 \mathrm{mM}-\mathrm{CaCl}_{2}$ structure factors with the solid curve in Figure 1 (a) shows that the membrane pair separation, $S$, remains very nearly constant when the water swelling is reversed in $1 \mathrm{~mm}-\mathrm{CaCl}_{2}$. The $1 \mathrm{~mm}-\mathrm{CaCl}_{2}$ Patterson function, Figure 2 (b), is nearly identical to that for water out to about $50 \AA$; beyond, there is a peak at the half period, $83 \AA$, in the one case but at $72 \AA$ in the other. Note that for myelin swollen in water there always is a Patterson peak at $D-72 \AA$ which is the mirror image, about $D / 2$ of the $72 \AA$ peak; therefore the peak at $D-72 \AA$ moves outward or inward as $D$ increases or decreases, i.e. as the water layer becomes thicker or thinner. In Figure 2(b) superimposed on the Patterson function for nerve in $1 \mathrm{mM}-\mathrm{CaCl}_{2}$ are two 
origin-like peaks, one $(\cdots \cdots)$ centered at $72.5 \AA$ and the other $(-\ldots--)$ at $D-72.5 \AA(93.5 \AA)$. The good fit of the algebraic sum $(\bigcirc \bigcirc \bigcirc \bigcirc \bigcirc)$ of the two peaks shows that the Palterson peak at $D / 2$ is the sum of two components which are not resolved.

The observed odd-order reflections of the pattern from the specimen in $1 \mathrm{mM}-\mathrm{CaCl}_{2}$ indicate asymmetry of the structure of a single membrane (see Schmitt, Bear \& Palmer, 1941). This Patterson function interpretation, which assumes bilayer membrane structure, indicates that the asymmetry is due to a largely symmetric membrane profile being located off-center within the half-period defined by extracellular and cytoplasmic interfaces.

\section{(c) Analysis of the normal myelin pattern: cyloplasmic swelling}

The square roots, $F_{h}$, of the corrected reflection intensities for normal myelin lie somewhat off the $F_{\mathrm{I}}$ curve for extracellular swelling (see Fig. 1(a)); there is no scale factor, $\alpha$, to make them fit. Therefore the pair of membranes in normal myelin has a structure somewhat different from that of the membrane pair in myelin swollen in water.

Adding together two components to account for the normal Patterson peak at $D / 2$, Figure 2(c), those need to be centered at $77.5 \AA$ and at $93.5 \AA(D-77.5 \AA)$ : assuming bilayer structure, the pair of membranes is $77.5 \AA$ apart, center to center across the cytoplasmic interface. In normal myelin $S$ is therefore $5 \AA$ greater than its value, $72 \cdot 5 \AA$, for nerve in $1 \mathrm{mM} \cdot \mathrm{CaCl}_{2}$. That $S$ increases by the same amount as the period, from 166 to $171 \AA$, indicates swelling at the membrane interface opposite to that for swelling in water, i.e. there is swelling at the cytoplasmic interfaces between adjacent membrane pairs. The cytoplasmic swelling is well controlled by the concentration of the salt solution, and consequently there is a means once again to explore an $F^{2}$ curve of a membrane pair. However, swelling at the cytoplasmic interface gives a different $F^{2}$ curve; the Fourier transform is denoted $F_{\text {II }}$ to distinguish it from $F_{\mathrm{I}}$, that for swelling at the extracellular interface.

For the cytoplasmic swelling the period varies by no more than $30 \AA$. Consequently the orders of diffraction in one set are never so closely spaced as for the extracellular swelling (periods to over $400 \AA$ ). As a result, intensities for the several periods do not cover all parts of the $F_{\mathrm{II}}^{2}$ curve. Nonetheless, connecting closely adjacent intensities with straight lines clearly shows slopes of the $F_{\mathrm{II}}^{2}$ curve.

By Geren's (1954) hypothesis of myelination one assumes that the profile of one of the membranes in a pair is the mirror image of the other. $F_{I}$ is therefore real, i.e. either positive or negative at each point. Now $F_{\mathrm{I}}^{2}$ is such that orders 2 and 3 of the $1 \mathrm{~mm}-\mathrm{CaCl}_{2}$ pattern must have the same algebraic sign (see Fig. 1(a)); similarly for orders 4 and 5 . Noting that $F_{\mathrm{I}}$ is the Fourier transform of a membrane pair centered at the cytoplasmic interface, whereas the pair for $F_{\text {II }}$ is centered at the extracellular interface, there is a simple relationship between the two transforms: in moving the crystallographic origin from the cytoplasmic to the extracellular interface each odd order changes sign while each even order keeps the same sign. Since, in moving the origin, the signs of orders 3 and 5 of the $1 \mathrm{mM}-\mathrm{CaCl}_{2}$ pattern both change while those of 2 and 4 remain the same, $F_{\mathrm{II}}$ is predicted to have a zero between orders 2 and 3 and another between orders 4 and 5 ; the slopes observed for the corrected intensities, $F_{h}^{2}$, of the normal and $\mathrm{I} \mathrm{mM}-\mathrm{CaCl}_{2}$ patterns indicated the zeros predicted in the $F_{\text {II }}$ curve (see Fig. 3). A peak in $F_{\mathrm{II}}^{2}$ was indicated between orders 


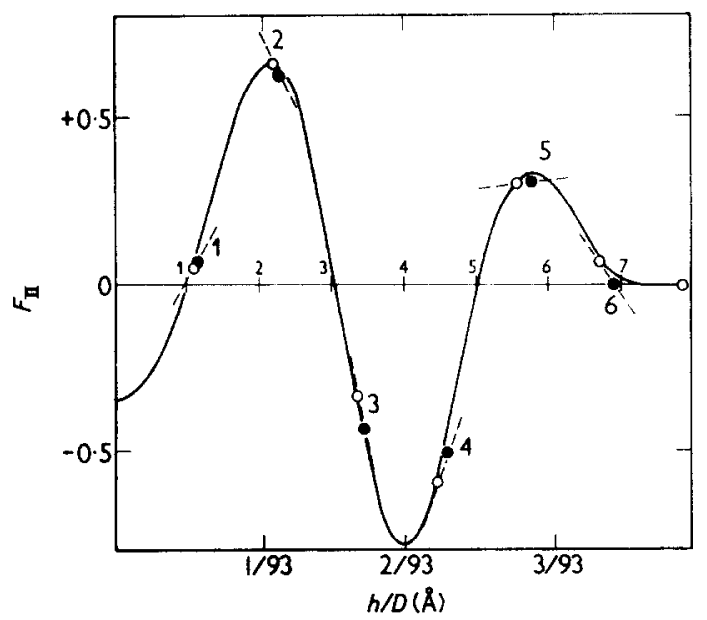

FIa. 3. The Fourier transform, $F_{\text {II }}$, derived from cytoplasmic swelling, when the crystallographic origin of the membrane pair is at the extracellular interface. $F_{h}$ is plotted versus $h / D$. The open circles (O) show orders of the normal pattern; the closed circles (O), of nerve in 1 mM-CaCl 2 . The numbered dashed lines, (- - - - ), passing through structure factors of the same index $h$, indicate the slopes of the Fourier transform $F_{11}$ while the solid line shows the best fitting reconstructed (Blaurock, 1967) Fourier transform. The numbered crosses $\left({ }^{h}+\right)$ show the positions of the orders of the $190 \AA$ period observed by Finean \& Millington (1957) for frog sciatic nerve in hypertonic Ringer's solution: the odd orders generally were observed to be weak, as predicted, confirming that these authors observed swelling at the cytoplasmic interface.

3 and 4 since the $F_{\text {II }}^{2}$ curve was rising at 3 and falling at 4 ; similarly, peaks were indicated between orders 1 and 2 and between orders 5 and 6 .

Comparing the two sets of intensities for nerves in 0.125 and $0.25 \mathrm{M}-\mathrm{LiCl}$ solutions (Blaurock, 1967), $D$ equal to 171 and $173 \AA$, respectively, the same slopes were found, order for order. Similarly for the sets of intensites for nerves in $0 \cdot 125$ and $0 \cdot 25 \mathrm{M}-\mathrm{CsCl}$ solutions, when again $D$ equals 171 and $173 \AA$, respectively.

When a nerve, the myelin of which had been allowed to swell in water, was put back into Ringer's solution, the myelin period, $D$, dropped from $250 \AA$ to a value a few $\AA$ units below normal. As recorded in a series of exposures, $D$ then gradually increased to the normal $171 \AA$ during the next few hours (Worthington \& Blaurock, $1969 a$ ), and at the same time the intensity distribution, which at first resembled that for nerve in $1 \mathrm{~mm}-\mathrm{CaCl}_{2}$, returned to normal. Thus as $D$ increased in the series of exposures, the intensity distributions conformed to the square of the $F_{\text {II }}$ curve shown in Figure 3.

In sum, when a freshly dissected nerve is put in water, each membrane pair moves away from the two adjacent pairs as broad (upwards of $80 \AA$ thick) layers of water form, and-at the same time-the two membranes in each pair move closer together, i.e. $S$ decreases by 5 to $6 \AA$. When the nerve is then put back into Ringer's solution, the broad layers of water disappear rather rapidly; more slowly, the two membranes in a pair move apart by 5 to $6 \AA$. The pattern is finally distinguishable from that of the freshly dissected nerve only in a very slight broadening of each reflection. The force causing broad water layers to form may be electrostatic (Worthington \& Blaurock, $1969 a$ ). The changes in membrane-pair separation, $S$, appear to reflect permeation of the membrane by salts (see section (f) of the Discussion). 

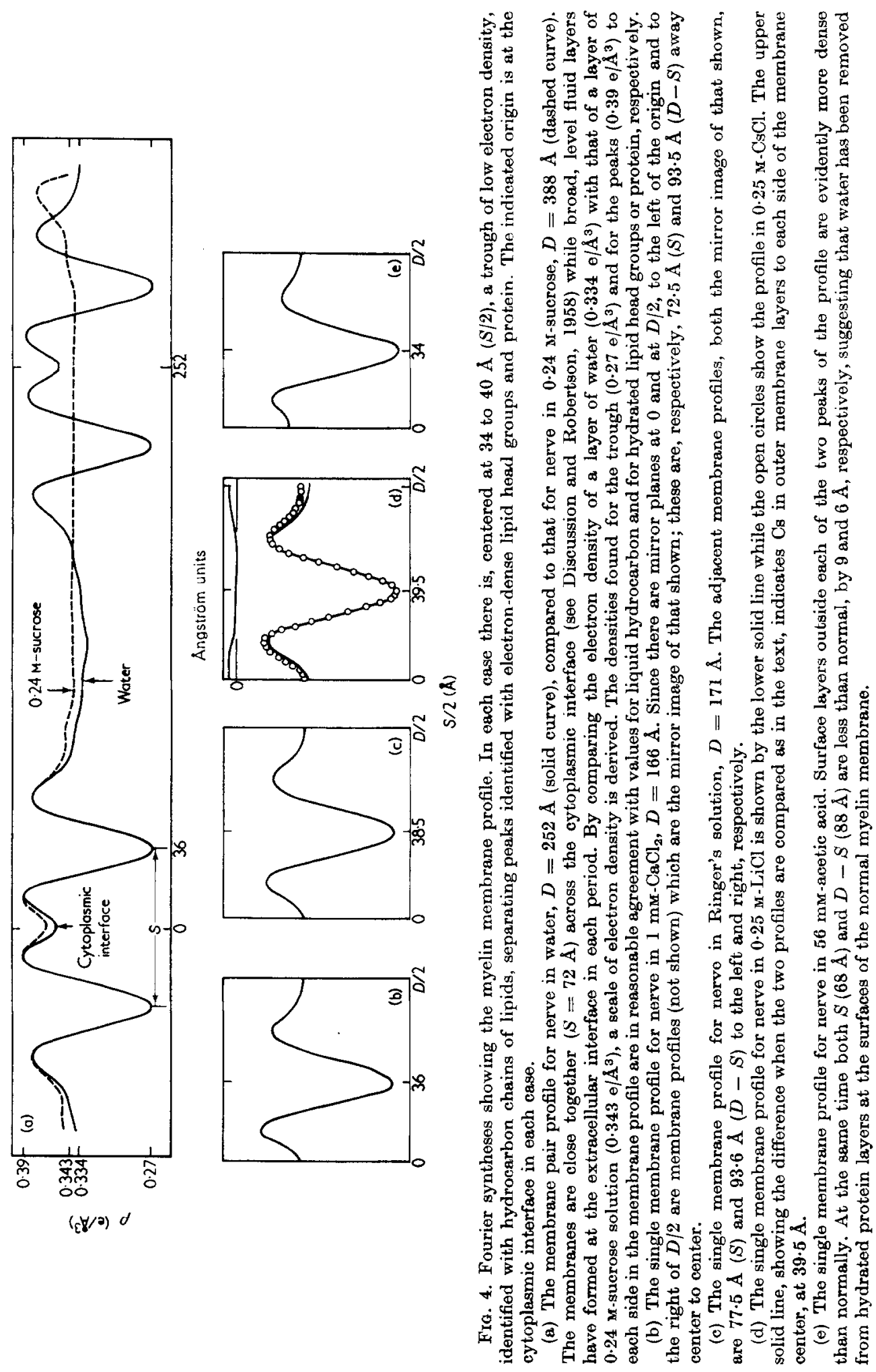


\section{(d) Fourier synthesis: the myelin membrane profile}

Fourier synthesis in accord with the Patterson function interpretation gives the profiles of the membrane pair, all to the same resolution, shown in Figure 4. In each case, to the right of the origin there is a bilayer membrane profile-a trough corresponding to a low-density layer of the lipid hydrocarbon chains separating two peaks corresponding to layers of electron-dense lipid head groups and protein. In each case the membrane pair center-to-center separation, $S$, is that given in Table 1 .

It is not surprising that the single membrane profiles in the curves shown in Figure $4(a)$ to (d), are nearly identical, for it is a condition of the existence of $F_{I}$ (Fig. l(a)) and $F_{\text {II }}$ (Fig. 3 ) that the structure of each single membrane does not change. It follows that the two kinds of swelling, both reversible, determine the membrane profile, for any other choice of algebraic signs for the orders of diffraction gives profiles which do not resemble each other as closely. For example, I have computed Fourier syntheses using our data $\left(\left|\boldsymbol{F}_{h}\right|\right)$ and the phasing derived by Akers \& Parsons (1970): the well-defined differences evident in comparing the single membrane profiles for the various experiments clearly are significant since the sets of data are all to the same resolution.

Thus the correctness of the profiles in Figure 4(a) to (d), is shown independently of the assumption, useful in interpreting the Patterson functions, that the single myelin membrane has bilayer profile. Rather, the less restrictive condition that the membrane structure changes little, if at all, under the mild and reversible experimental treatments of the myelin is met, as one confirms by inspecting the membrane profiles shown. The Patterson analysis serves to derive the single membrane profile, but its correctness is demonstrated crystallographically.

\section{(e) A scale of electron density for the bilayer profile}

For pairs of membrane separated by broad, uniform layers of the test solution from adjacent pairs, there will be reflection intensity changes as the electron density of the test solution is varied. When the two $F_{\mathrm{I}}$ curves for two test solution densities are compared, the predicted difference term (Blaurock, 1967) is everywhere proportional to the change in the electron density of the layers of solution; the difference term (the open circles in Fig. $1(\mathrm{c})$ ) is largest nearest the center of the pattern and shows oscillations attenuating rapidly with increasing distance from the center. Since its form is everywhere known, it can in principle be used to find the algebraic signs needed for a Fourier synthesis.

Comparing, in the light of prediction, the innermost myelin reflections from nerve in water and in the three different sucrose solutions, region $A$ in Figure $1(\mathrm{c})$, the intensities - the squares of the distances from the horizontal axis-show a pattern of changes: fairly intense in water, weakest in $0.24 \mathrm{M}$-sucrose, intense again in 0.82 M-sucrose and most intense in $1.80 \mathrm{M}$-sucrose. Since a match of test solution to the average membrane pair density will give an $F_{\text {I }}$ curve approaching zero at the origin (Blaurock, 1967), the membrane pair average electron density is closest to that of $0.24 \mathrm{M}$-sucrose. Consequently the algebraic signs of the first order for nerve in water and of the first two orders for $0.82 \mathrm{M}$-sucrose and $1.80 \mathrm{M}$-sucrose are evident: positive in water and negative in the concentrated sucrose solutions (see Fig. 1(c)). As predicted, the differences in $F_{I}$ for the solutions of different electron densities fall away rapidly. However, other differences are apparent in Figure 1(c): these differences 
clearly indicate a change in the membrane profile, possibly because sucrose permeating to the cytoplasmic interface increases the density there (see section (f) of the Discussion).

The changing electron density of the swelling solution is evident when profiles are compared. The two Fourier syntheses of swollen myelin, Figure 4(a), show broad level regions which are identified with uniform fluid layers; the slight ripple is evidently the effect of finite resolution (observed profile diffraction beyond $27 \AA$ has been omitted). Superimposing the membrane profile for myelin in water (- - ) on the membrane profile for myelin in $0.24 \mathrm{M}$-sucrose (- - - - the assumption is implicit that sucrose does not contribute at the centers of the two bilayer peaks (see section (e) of the Discussion)-and assuming that the level regions are broad layers of the test solutions, the calculated electron densities of which are given in Figure 4(a), one has an absolute scale of electron density. Taking into account the limited resolution (which is the same for all the profiles in Fig. 4) the electron densities found for the bilayer trough and for the peaks to each side compare satisfactorily to the respective values expected for a liquid hydrocarbon-like layer $\left(0.30 \mathrm{e} / \AA^{3}\right.$; Rand \& Luzzati, 1968) and for a lipid head-group or protein-like layer $\left(0.45 \mathrm{e} / \AA^{3}\right.$ for anhydrous protein; Blaurock, 1967). The bilayer profile is firmly established.

\section{Discussion}

\section{(a) Proof of the membrane profile}

By analyzing, in terms of bilayer structure, experiments in which the myelin lowangle period and reflection intensities vary reversibly, a profile of the single membrane has been derived. Then, ignoring the bilayer assumption and using only the wellconfirmed condition that the single membrane structure is substantially unchanged as the center-to-center spacings of membranes are varied, the bilayer profile, very nearly identical in each case in Figure 4(a) to (d), is confirmed. For no other phasing is there a constant membrane profile. For example, an alternative phasing (Akers \& Parsons, 1970) for the Fourier syntheses shows membrane profiles which differ significantly for the various cases. Thus the Patterson function analysis of the swelling experiments leads to a profile for the single myelin membrane, but the correctness of the bilayer profile is demonstrated crystallographically in Figure 4.

(b) Other modifications of the myelin sheath structure

The structure of the myelin sheath has been modified in other ways (Blaurock, 1967). When a fresh nerve was soaked in Ringer's solution made $0.25 \mathrm{~m}$ in sucrose, a pattern with the normal period, but with a distribution of intensities somewhat different from the normal (leading to the slightly lower value of $S$ in Table 2), was observed as long as the solution was kept cold (at $4^{\circ} \mathrm{C}$ for 3 days; exposed at room temperature for $1 \mathrm{hr}$ ). The specimen was then kept at room temperature, and the period increased within 13 hours to $188 \AA$ while the Patterson function analysis indicated swelling at the extracellular interface (see $S$ in Table 2). Bilayer profiles closely similar to those in Figure 4(a) to (d), were seen in the two computed Fourier syntheses.

For a frog sciatic nerve soaked first in water and then in 56 mm-acetic acid, $\mathrm{pH} 3$, the period was $156 \AA$. The Patterson analysis showed the value of $S$ had dropped to $68 \AA$ (Table 2 ). In this case, there is evidently a significant increase in the electron density of the outer layers of the membrane profile (see Figure 4(e)).

After the more extreme treatments of freezing the nerve, or drying it, or soaking 


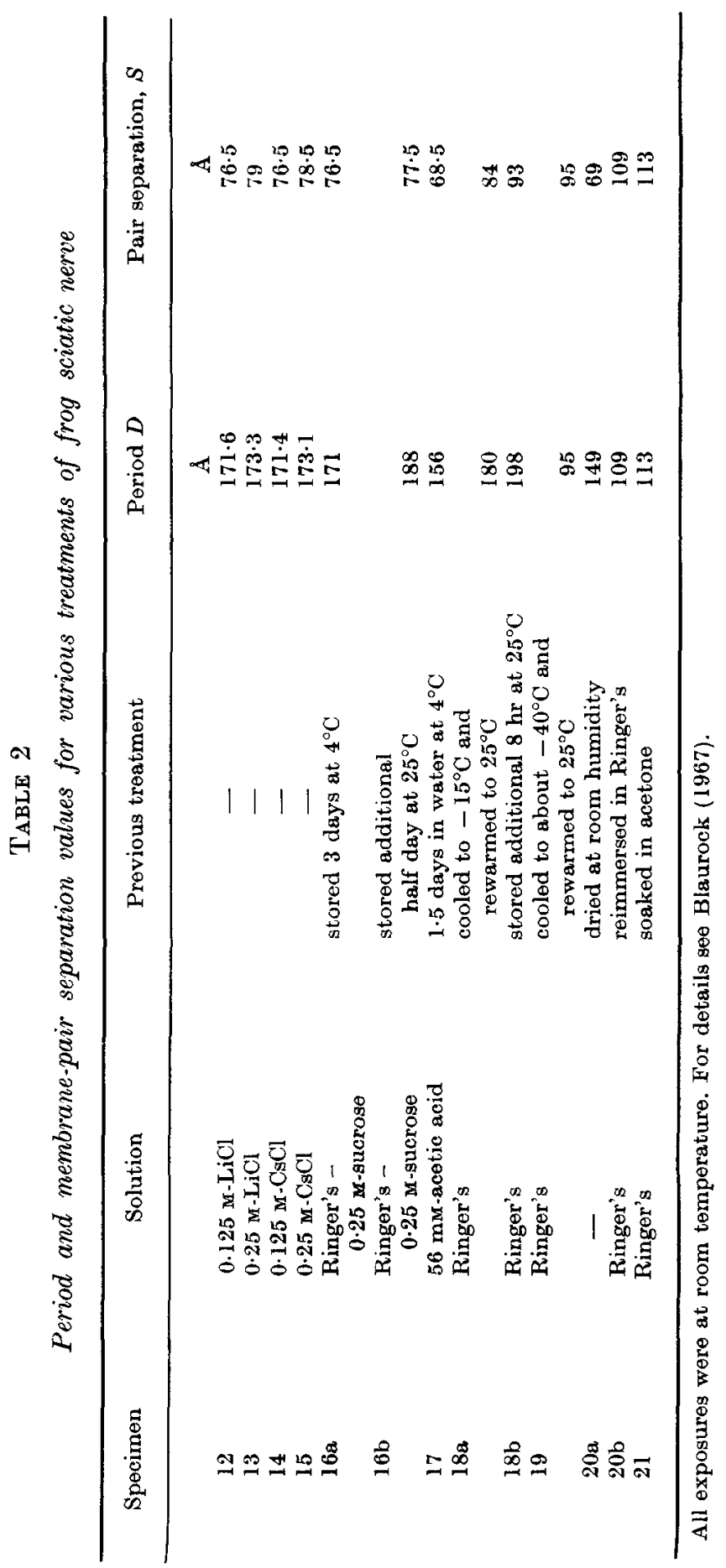


it in acetone (see Fernández-Morán \& Finean, 1957), and then returning it to Ringer's solution, extensive changes were seen in the period and intensities (see values of $D$ and $S$ in Table 2). Nonetheless, a similar bilayer profile was evident in each of the Fourier syntheses computed to the same resolution $(H / D \cong 1 / 28 \AA)$ according to the Patterson function interpretation. In as much as similar bilayer profiles can be demonstrated for a variety of experimental modifications of the myelin sheath, there can be little doubt of the correctness of the bilayer profile in Figure 4.

\section{(c) Comparison with other myelinated nerves}

The Patterson functions calculated from the low-angle diffraction patterns observed for peripheral and central nervous system myelin of various animals (Blaurock \& Worthington, 1969) have been analyzed in the same way (Blaurock, 1967). The results indicate that, to the present resolution, the myelin membrane profile in each case is a largely symmetric bilayer similar to that of frog sciatic myelin.

\section{(d) Scaling errors}

The formula used to scale patterns (see Methods) involves an approximation in that $F_{0}^{2}$ is unobserved and so must be taken as zero in the first instance. In general, omitting $F_{0}^{2}$ will have resulted large scaling errors neither in the scaling of data sets in Figures 1(a), (b) and 3 nor in the analysis of the Patterson functions.

For example, a simple calculation shows that omitting $F_{0}^{2}$ in comparing the several sets of water swelling data, Figure $1(a)$, introduces a relative scaling error not exceeding $7 \%$ as compared to taking for $F_{0}$ the value +0.5 . Similarly, in comparing the several sets of $0.24 \mathrm{M}$-sucrose data the relative scaling error does not exceed $0.1 \%$ as compared to taking the value $+0 \cdot 1$ for $F_{0}$. These errors, which are no more than comparable to errors in measuring film density, therefore present no serious difficulty.

Aside from numerical considerations, Figure 1 clearly shows that a satisfactory scaling has been made. No more need be asked of the working method. To suppose that the relative scaling of any two sets of data in Figure 1(a) or 1(b) or 3 is largely in error would be to substitute a complicated interpretation for a simple one which has been shown to encompass a number of experiments.

Omitting $F_{0}^{2}$ from the computed Patterson function clearly will generally shift the $70 \AA$ Patterson peak downward relative to the origin peak, the more so the greater the mismatch between the pair average density and the fluid density; at the same time the half-width of the $70 \AA$ peak will be undervalued. Using the value found for $F_{0}$ (Fig. 1 (a)) in computing the Patterson function of nerve in water, the $72 \AA$ peak height will be $54 \%$ of the origin peak height rather than the $50 \%$ given in Table 1 (when $F_{0}^{2}$ is omitted). As a second example, the height of the $78 \AA$ peak for nerve in $1.80 \mathrm{M}$-sucrose will be $52 \%$ of the origin peak height rather than the $45 \%$ given in Table 1. Such errors are comparable to or perhaps slightly exceed the error that will arise due to error in measuring film density. This error cannot significantly affect the fitting of the origin-like peaks to the half period peak in Figure 2(b) or 2(c) since these fits will be as good as that in Figure 2(a) - a point borne out by the agreement between predicted values of $S$ with those measured on the profiles of Figure 4.

\section{(e) Single membrane average electron density}

The predicted effect on the reflection intensities of varying the electron density of the solution in which myelin swells has been demonstrated: a large systematic 
variation is observed in the intensities of the innermost reflections. From this variation, which near the origin is of the form $\sin (\chi) / \chi$ with $\chi=[\pi \cdot 166 \AA \cdot(h / D)]$ (Fig. $1(\mathrm{c}))$, an average electron density of the myelin membrane pair of $0.347 \mathrm{e} / \AA^{3}$ is calculated, a value somewhat greater than the $0.343 \mathrm{e} / \AA^{3}$ of $0.24 \mathrm{M}$-sucrose. Values found for the electron density of the trough and of the adjacent peaks in the profile of the single membrane are consistent with a lipid bilayer at the center of the membrane. A lipid bilayer will, of course, account for the hydrocarbon chains orientation deduced by Schmitt et al. (1935). At the same time it is not excluded that some protein protrudes into the bilayer.

Both because of the limited data recorded and the rather considerable extrapolation to the levels of the peaks and trough of the single membrane profile, the scale of absolute electron density in Figure 4(a) cannot be presumed to be of high accuracy. Nonetheless, levels of the fluid layers are likely to be shown with good accuracy relative to the average level of the membrane proper. This is so because the correction (see Methods) to the observed intensities, $I_{h}$, means the data in region A in Figure 1, which largely determine the relative level, are unusually accurate relative to the data in regions $\mathrm{B}$ and $\mathrm{C}$.

Assuming an insignificant amount of sucrose at the center of each of the two bilayer peaks in the single membrane profile of nerve in $0.24 \mathrm{M}$-sucrose is unlikely to have introduced a large scaling error since the central membrane trough, which corresponds to the lipid hydrocarbon chains of a bilayer and so is assumed not to contain much sucrose, superimposes well on the trough for nerve in water. If considerable sucrose were present at the center of the membrane peaks, the electron density found for these would be lower. The value found is, in fact, thought not to be overly large in view of the apparently well-defined layer of lipid head groups responsible for each of the two peaks (Wilkins et al., 1971). In the present study the accuracy in determining absolute electron densities on the profile is thought to be limited most severely by the comparatively coarse resolution (see section $(g)$ below).

\section{(f) The nature of the swelling of frog sciatic myelin}

The swelling apart of membrane pairs when myelin is in water has been shown not to be osmotic (Worthington \& Blaurock, 1969a). Rather, myelin extracellular swelling does not occur, in general, when there is a sufficiently concentrated salt solution bathing the nerve; the required concentration depends on the cation. The presence of the cation in the unswollen sheath is shown in Figure 4(d), where the membrane profiles of nerve in $0.25 \mathrm{~m}-\mathrm{LiCl}(-)$ and $\mathrm{CsCl}(00000)$ solutions are compared. Assuming, in order to superimpose the one profile on the other, that none of the more electron-dense $\mathrm{Cs}$ is in the central hydrocarbon layer and that the $\mathrm{CsCl}$ profile will not lie below the $\mathrm{LiCl}$ profile at any point, Figure 4(d) shows layers of Cs at both the cytoplasmic and extracellular surfaces of each membrane. When spread over 30 A -wide layers the amount of $\mathrm{Cs}$ necessary to give the small increase observed over the $\mathrm{LiCl}$ profile (see upper solid curve, Fig. 4 (d)) is at a concentration about the same as that in the bulk solution. Although Cs evidently replaced $\mathrm{Li}$ isomorphously, the intensity changes are rather small to permit phasing without strong assumptions. Nonetheless, the comparison indicates that each membrane in the pair profile has aqueous surface layers.

The degree of extracellular swelling was not repeatable. Rather, the swollen period varied from 250 to $340 \AA$ in water; from 290 to $390 \AA$ in $0.24 \mathrm{M}$-sucrose. That there 
tended to be a larger period in the sucrose solutions as compared to water may reflect osmotic swelling and shrinking of the nerve axon and Schwann cell cytoplasm within the space defined by the endoneurium, a collagenous sheath surrounding each myelinated nerve fiber (Worthington \& Blaurock, 1969b). A freshly dissected nerve put directly into 0.24 M-sucrose swelled only to $293 \AA$, the lowest period for this concentration: by omitting the water soaking, when cytoplasmic salts will be leached out of these cells, there may have been less space made available to the expanding myelin.

The kind of swelling evident in Figure 3 is, in contrast to the swelling evident in Figure 1, closely controlled by the concentration of the salt solution. It has been considered whether this swelling may be due to salt permeating the myelin membrane. When a freshly dissected nerve is placed in water, there will be a net efflux as salts in the cytoplasmic space cross the membrane to reach the extracellular space of the myelin. Assuming that the hydration of the membrane protein and lipids does not change, adjacent membranes will move together due to the volume of these salts and their water of hydration lost from the cytoplasmic space. Similarly, when the swollen nerve is put back into Ringer's solution the net influx of salts will swell the membranes apart again, thus accounting for the observed gradual increase of centerto-center spacing, $S$, of the membrane pair. Taking a cytoplasmic layer of the order of $10 \AA$ wide and a permeability constant of $1 \times 10^{-8} \mathrm{~cm} / \mathrm{sec}$ (squid axon membrane; Brinley \& Mullins, 1965), equilibrium will be reached in seconds. The observed time-course, several hours, suggests a permeability constant three orders of magnitude lower.

If the swelling shown in Figure 3 is by the permeation hypothesized, then this swelling clearly is at the cytoplasmic interface while the swelling shown in Figure 1 is consequently at the extracellular interface of the myelin membranes. In agreement with this interpretation Robertson (1958) has observed in electron micrographs that swelling in hypotonic Ringer's solution is at the extracellular interface.

Finean \& Millington (1957) reported that myelin swells reversibly to a $190 \AA$ period in Ringer's solution ten times the normal concentration. For cytoplasmic swelling one predicts from the $F_{\text {II }}$ curve, Figure 3 , that the odd-order intensities will be quite small. The tracing published by these authors shows prominent even orders $(2,4,6)$ while of the odd orders only a rather small first is apparent. It is therefore reasonable to suggest this is a case of swelling at the cytoplasmic interface. Using $7 \cdot 7$ times normal Ringer's solution we confirmed their result, observing a $185 \AA$ period (Worthington \& Blaurock, 1969a) and weak odd-order reflections.

The value of $1 \times 10^{-8} \mathrm{~cm} / \mathrm{sec}$ has been reported (Mullins, 1966; Ussing, 1966; Collander \& Bärlund, 1933) for sucrose permeating various membranes; again the time to reach equilibrium is seconds. (A permeability constant three orders of magnitude lower (for asolectin vesicles; Miyamoto, personal communication) would still allow equilibrium to be reached within the time of the experiment.) A comparison of the membrane pair profiles of myelin in water and in $0 \cdot 24 \mathrm{M}$-sucrose, Figure $4(\mathrm{a})$, suggests that sucrose permeating the membranes accounts for the increased density at the interface within the membrane pair. However, only in the case of 1.80 M-sucrose did the membrane pair spacing, $S$, increase, to $78 \AA$. Assuming that the increased density on the pair profile shows sucrose, the failure to see the membranes move apart when the nerve is taken from water and bathed in 0.24 or $0.82 \mathrm{M}$-sucrose suggests that the sucrose displaces its own volume in water from the cytoplasmic 
space. Sucrose present in the cytoplasmic space will account for the positive (upward) shift of $F_{\mathrm{I}}$ (see Fig. 1 ) as the concentration of sucrose increases.

\section{(g) Details of the membrane structure}

In Figure 4(a), layers significantly more dense than water are apparent on the extracellular surface of each membrane, outside the more or less symmetric dense peak. That there may in fact be hydrated layers of protein on both surfaces of the single membrane is supported on the one hand by the apparent penetration of sucrose (Fig. 4(a)) or Cs (Fig. 4(d)) up to the dense peak on each side, and on the other hand by the increased density of these surface layers when the membranes move closer together as in the acetic acid solution (Fig. 4(e)), in which case both $S$ and $D-S$ decrease from the normal (from 78 to $68 \AA$ and from 93 to $88 \AA$, respectively).

More detail will be apparent in the membrane profiles when observed reflections (Blaurock \& Worthington, 1969) beyond the six used to compute the normal myelin profile (Fig. 4(c)) have been included. Because the diffraction beyond $28 \AA$ is generally weaker than the lowest orders, these higher orders will not change the basic profile. Rather they will add detail.

The intensity distribution of the higher orders $(h>6)$ gives a clue, as follows, to the correct phasing. The two Fouricr transform pcaks, regions $\mathrm{B}$ and $\mathrm{C}$ in Figure 1 , are the result of interference between the two membranes in one pair; the transform of the single membrane is a broad peak extending over both regions. If the bilayer membrane profile is symmetric in detail, then more pair interferences will be apparent in the intensity distribution of the higher orders of the normal pattern; the regular spacing of the intensity peaks will correspond to the pair separation, S. Similarly, interferences corresponding to the bilayer peaks in one membrane, which are $45 \AA$ apart, center to center, will also be evident (Wilkins et al., 1971). These two effects are recognized, and a higher resolution profile has been computed (Blaurock \& Wilkins, manuscript in preparation).

I am grateful to Professor C. R. Worthington for support and for the use of his laboratory facilities in the Biophysics Division of the Institute of Science and Technology at the University of Michigan, Ann Arbor, and to Professor M. H. F. Wilkins for useful discussions during the tenure of a National Institutes of Health postdoctoral fellowship at the Biophysics Department, King's College, London. I thank Drs D. M. Engelman, V. K. Miyamoto and D. Oesterhelt for their helpful criticisms of the manuscript. Grateful acknowlodgement is made for support through a National Science Foundation Co-operativo Graduate Fellowship during part of this work.

\section{REFERENCES}

Akers, C. K. \& Parsons, D. F. (1970). Biophys. J. 10, 116.

Blaurock, A. E. (1967). Ph.D. Thesis, University of Michigan.

Blaurock, A. E. \& Worthington, C. R. (1966). Biophys. J. 6, 305.

Blaurock, A. E. \& Worthington, C. R. (1969). Biochim. biophys. Acta, 173, 419.

Brinley, F. J. \& Mullins, L. J. (1965). J. Neurophysiol. 28, 526.

Collander, R. \& Bärlund, H. (1933). Acta Bot. F'enn. 11, 1.

Fernández-Morán, H. \& Finean, J. B. (1957). J. Biophys. Biochem. Cytol. 3, 725.

Finean, J. B. \& Burge, R. E. (1963). J. Mol. Biol. 7, 672.

Finean, J. B. \& Millington, P. F. (1957). J. Biophys. Biochem. Cytol. 3, 89.

Geren, B. B. (1954). Exp. Cell Res. 7, 558.

Moody, M. F. (1963). Science, 142, 1173.

Mullins, L. J. (1966). Ann. N.Y. Acad. Sci. 137, 830. 
Rand, R. P. \& Luzzati, V. (1968). Biophys. J. 8, 125.

Robertson, J. D. (1958). J. Biophys. Biochem. Cytol. 4, 349.

Schmitt, F. O., Bear, R. S. \& Clark, G. L. (1935). Radiol. 25, 131.

Schmitt, F. O., Bear, R. S. \& Palmer, K. J. (1941). J. Cell. Comp. Physiol. 18, 31.

Ussing, H. H. (1966). Ann. N.Y. Acad. Sci. 137, 543.

Wilkins, M. H. F., Blaurock, A. E. \& Engelman, D. M. (1971). Nature, in the press.

Worthington, C. R. \& Blaurock, A. E. (1969a). Biochim. biophys. Acta, 173, 427.

Worthington, C. R. \& Blaurock, A. E. (1969b). Biophys. J. 9, 970.

Note added in proof: I thank $\mathrm{D}$. L. D. Caspar and D. A. Kirschner for their kindness in sending me a copy of a manuscript on X-ray diffraction analysis of myelin membrane structure, which they have submitted to Nature. They offer an alternative proof for the bilayer structure of the rnembrane and confirm my conclusion that the asymumetry apparent from the low-angle diffraction pattern is explained by a largely symmetric profile located eccentrically in the space of one half-period. Their profile, which is to a higher resolution than those shown in Figure 4, is interpreted by them as showing an asymmetrio arrangement of the cholesterol present. 